\title{
INTERFERENCE EXPLOITATION-BASED HYBRID PRECODING WITH ROBUSTNESS AGAINST CHANNEL ERRORS
}

\author{
Yufan Fan*, Ganapati Hegde*, Christos Masouros ${ }^{\dagger}$, Marius Pesavento* $^{*}$ \\ *Communication Systems Group, Technische Universität Darmstadt, Germany \\ ${ }^{\dagger}$ Department of Electrical and Electronic Engineering, University College London, UK
}

\begin{abstract}
The extremely high cost associated with massive multipleinput multiple-output (MIMO) systems when it is employed with fully digital precoding can be reduced by applying hybrid precoding at an expense of increased transmit power. In such a hybrid precoding system, the transmit power required to achieve a certain quality-of-service (QoS) can be significantly reduced by employing the constructive interference $(\mathrm{CI})$ precoding technique. However, as illustrated in the paper, the symbol error rate (SER) performance of CI-based precoding is very sensitive to channel errors. To address this challenge we propose a hybrid precoding approach with robustness against channel quantization error and channel estimation error. Simulation results demonstrate the superior energy efficiency of the proposed robust hybrid precoding when compared to that of a conventional non-robust precoding scheme in achieving a required QoS target.
\end{abstract}

Index Terms - massive MIMO, hybrid precoding, optimization, robust precoding, imperfect channel knowledge.

\section{INTRODUCTION}

When fully digital precoding architecture is employed in a massive multiple-input multiple-output (MIMO) system, the associated hardware costs are high [1-3], as each antenna requires a dedicated radio-frequency (RF) chain. Hybrid precoding is one of the solutions proposed in the literature to reduce the hardware cost in massive MIMO systems [4-7], where fewer RF chains are employed as when compared to the fully digital precoding architecture. In the hybrid precoding, analog phase shifters are used for the analog precoding and are connected with the digital precoding through a limited number of RF chains. Instead of adjusting both magnitude and phase of the transmitted symbols as in the digital precoding, in analog precoding only the phase of the incoming signal is adapted, which leads to a reduced degree of freedom in the precoder design. As a consequence, the required transmit power is increased to achieve the same QoS, in contrast to fully digital precoding [8-10]. One approach to improve the power efficiency is using the constructive interference (CI)

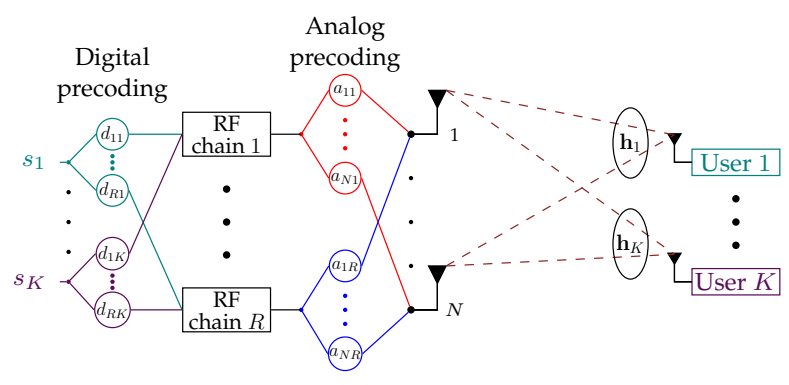

Fig. 1. Schematic diagram of the hybrid analog-digital precoding system architecture (image source [11]).

technique, in which the interference power is exploited to improve the useful signal power at the users [11-16].

In numerous $5 \mathrm{G}$ communication scenarios, e.g., vehicleto-x communication, remote medical operations, drone delivery, etc., the communication systems need to be robust against multiple factors such as interference, imperfect channel knowledge, hardware impairments [17-19]. In [11], impairments in the analog phase shifters of hybrid precoding architecture are considered and a corresponding optimal robust precoding technique is proposed. In this work, we extend this approach into hybrid precoding massive MIMO systems with robustness against two possible types of channel errors, namely, quantization errors and estimation errors.

\section{SYSTEM MODEL}

Consider a co-channel multi-user massive MIMO downlink system as depicted in Fig. 1. It consists of a base station (BS) equipped with $N$ transmit antennas and $R$ RF chains, where $R<N$. Let $\mathcal{K} \triangleq\{1, \ldots, K\}$ denote a set of $K$ singleantenna users served by the BS. The transmit symbol vector is given by $\mathbf{s} \triangleq\left[s_{1}, \ldots, s_{K}\right]^{\top}$, where the element $s_{k}$ indicates the symbol intended for the $k$ th user. The symbols are assumed to be drawn from an $M$-PSK constellation and without loss of generality, each transmit symbol is assumed to be of constant unit modulus. A digital precoder $\mathbf{d}_{k} \in \mathbb{C}^{R}$ is applied to the transmit symbol $s_{k}$ and the resulting signals are fed to the $R \mathrm{RF}$ chains. Each RF chain is connected to 


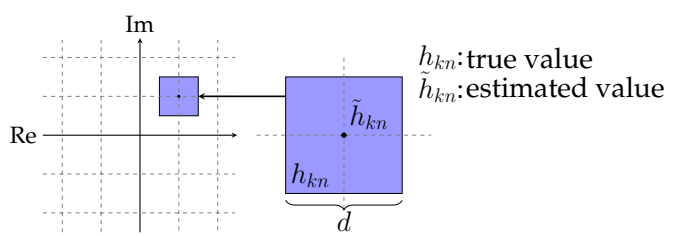

Fig. 2. Channel quantization error model with quantization step $d$.

all transmit antennas through analog phase shifters, which have constant gains. Let $\boldsymbol{a}_{r} \triangleq\left[a_{1 r}, \ldots, a_{N r}\right]^{\top}$ be the ana$\log$ precoder applied to the output of the $r$ th RF chain for $r \in \mathcal{R} \triangleq\{1, \ldots, R\}$. The matrix $\mathbf{A} \triangleq\left[\boldsymbol{a}_{1}, \boldsymbol{a}_{2}, \ldots, \boldsymbol{a}_{R}\right]$ denotes the analog precoding matrix.

Let $\mathbf{h}_{k} \in \mathbb{C}^{N}$ be the frequency-flat channel vector between the BS and the $k$ th user, and $n_{k} \sim \mathcal{C N}\left(0, \sigma_{k}^{2}\right)$ be the i.i.d. additive white Gaussian noise (AWGN) at the $k$ th user. The received signal $y_{k}$ at the $k$ th user can be expressed as

$$
y_{k}=\mathbf{h}_{k}^{\top} \mathbf{A}\left(\sum_{\ell=1}^{K} \mathbf{d}_{\ell} s_{\ell}\right)+n_{k} .
$$

The design of precoders typically assumes perfect knowledge of the channel state information (CSI) [20-22]. However, the CSI available at the transmitter is typically inaccurate due to limited resolution of the associated hardware devices, noise, environmental changes in practice. In FDD systems, the channel coefficients are typically estimated at the receivers, quantized, and fed back to the BS in the uplink [23-25]. Consequently, two potential stages may introduce errors, i.e., the estimation procedure and the quantization.

One typical source of error comes from the quantization procedure when applying the codebook mechanism, which is used to reduce the amount of feedback data from the users to the BS. The quantization error is for example depicted as in Fig. 2, where the quantized channel coefficient $\tilde{h}_{k n}$ is depicted in the center of the blue-colored square. The true channel coefficient falls anywhere in the blue-colored square and is related to the quantized channel by $h_{k n}=\tilde{h}_{k n}+e_{k n}$, with $e_{k n}$ indicating the channel quantization error. Let $\mathcal{E}$ denote an infinite set of all possible error vectors that are associated with the channel vectors, i.e.,

$$
\begin{array}{r}
\mathcal{E}=\left\{\mathbf{e}_{k}\left|\mathbf{e}_{k} \in \mathbb{C}^{N},\right| \operatorname{Im}\left(e_{k n}\right)\left|\leq \frac{d}{2},\right| \operatorname{Re}\left(e_{k n}\right) \mid \leq \frac{d}{2},\right. \\
\forall k \in \mathcal{K}, \forall n \in \mathcal{N}\} .
\end{array}
$$

Another type of channel error originates from the estimation procedure [26]. The channel state information is generally estimated by employing estimation methods, such as maximum-a-posteriori (MAP) estimation, minimum mean square error (MMSE) estimation [27]. The estimated channel coefficients typically suffer from errors when compared to the true channel coefficients in the environments. In this paper, we assume that the true channel coefficient $h_{k n}$ is confined

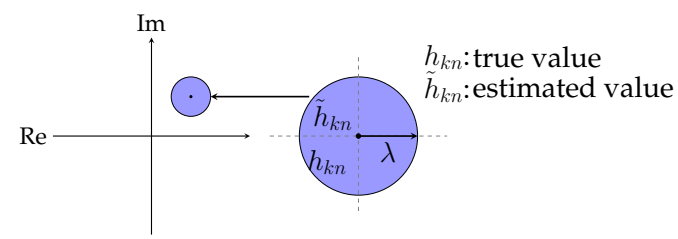

Fig. 3. Channel estimation error model with circle radius $\lambda$.

within a circle with center at $\tilde{h}_{k n}$ and radius $\lambda$ as depicted in Fig. 3. Define $\mathcal{G}$ as the infinite set of all the possible error vectors that are associated with the channel vectors, i.e.,

$$
\mathcal{G}=\left\{\mathbf{e}_{k}\left|\mathbf{e}_{k} \in \mathbb{C}^{N},\right| e_{k n} \mid \leq \lambda, \forall k \in \mathcal{K}, \forall n \in \mathcal{N}\right\} .
$$

\section{ROBUST HYBRID PRECODING}

In CI-based hybrid precoding, transmit signals are precoded such that the received signal at each user lies in the appropriate CI-region, which is a part of the decision region that is a threshold-margin $\Gamma$ away from the corresponding decision boundaries. ${ }^{1}$ In this paper, we consider the problem of computing the CI-based hybrid precoding that minimize the transmit power at the BS, such that the received signal at each user lies in the CI-regions of the corresponding transmit symbol. A corresponding optimization problem can be formulated as a semi-infinite program, given by [11]

$$
\begin{aligned}
& \min _{\mathbf{A},\left\{\mathbf{d}_{k}\right\}_{k \in \mathcal{K}}}\left\|\mathbf{A} \sum_{k=1}^{K} \mathbf{d}_{k} s_{k}\right\|^{2} \\
& \text { s.t. }\left|\operatorname{Im}\left(s_{k}^{*}\left(\tilde{\mathbf{h}}_{k}+\mathbf{e}_{k}\right)^{\top} \mathbf{A} \sum_{\ell=1}^{K} \mathbf{d}_{\ell} s_{\ell}\right)\right| \leq \\
&\left(\operatorname{Re}\left(s_{k}^{*}\left(\tilde{\mathbf{h}}_{k}+\mathbf{e}_{k}\right)^{\top} \mathbf{A} \sum_{\ell=1}^{K} \mathbf{d}_{\ell} s_{\ell}\right)-\gamma_{k}\right) \tan \theta, \\
& \forall k \in \mathcal{K},
\end{aligned}
$$

where $\theta \triangleq \pi / M$ denotes the angular distance between the transmit symbol and the corresponding decision boundaries for the modulation order $M$. The QoS controlling parameter $\gamma_{k} \triangleq \Gamma_{k} / \sin \theta$ with $\Gamma_{k}$ indicates the threshold-margin at the $k$ th user. The vector $\mathbf{e}_{k}$ is the channel error vector from the set $\mathcal{E}$ or $\mathcal{G}$ defined in Eqs. (2) and (3), respectively. The operators $(\cdot)^{*}$ and $(\cdot)^{\top}$ denote the complex conjugate and transpose function, respectively. Problem (4) can be reformulated as an equivalent single-group multicast problem as $[11,14,28]$

$$
\begin{aligned}
\min _{\mathbf{A}, \mathbf{b}} & \|\mathbf{A} \mathbf{b}\|^{2} \\
\text { s.t. } & \left|\operatorname{Im}\left(s_{k}^{*}\left(\tilde{\mathbf{h}}_{k}+\mathbf{e}_{k}\right)^{\top} \mathbf{A b}\right)\right| \leq \\
& \left(\operatorname{Re}\left(s_{k}^{*}\left(\tilde{\mathbf{h}}_{k}+\mathbf{e}_{k}\right)^{\top} \mathbf{A b}\right)-\gamma_{k}\right) \tan \theta, \forall k \in \mathcal{K} .
\end{aligned}
$$

\footnotetext{
${ }^{1}$ Due to space limitation, we refer readers to [11] for geometric visualization of the CI-region.
} 


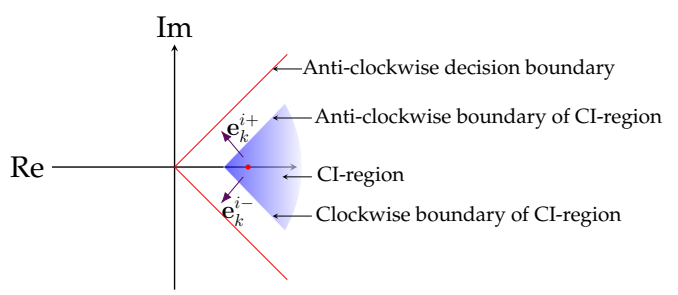

Fig. 4. Anti-clockwise and clockwise drifts of the received signals from the CI-region (image source [11]).

The optimal multicast digital precoder $\mathbf{b}^{\star}$ of the problem (5) and the optimal digital precoders $\mathbf{d}_{k}^{\star}$ of the problem (4) are related by $\mathbf{d}_{k}^{\star}=\mathbf{b}^{\star} / K s_{k}, k \in \mathcal{K}[11,14]$.

The optimization problem (5) is nontrivial to solve due to the following reasons. Firstly, the analog precoding matrix $\mathbf{A}$ and the digital precoder $\mathbf{b}$ are bilinear coupled, which makes the problem bilinear [29]. Such bilinear problem can be decomposed into analog precoding and robust digital precoding, where the robust digital precoder can be computed after the analog precoding matrix $\mathbf{A}$ is fixed to $\hat{\mathbf{A}}$ [11]. Numerous analog precoding techniques can be applied in hybrid precoding systems, such as conjugate phase of channel (CPC) method and best matching code selection (BMCS) method [30,31]. Secondly, the optimization problem has an infinite number of constraints. To address this problem, we extend the iterative algorithm proposed in [11] to the current problem with channel errors. The algorithm is initialized with $\mathcal{E}_{k}^{i+}=\{\mathbf{0}\}$ and $\mathcal{E}_{k}^{i-}=\{\mathbf{0}\}$ for the first iteration $(i=1)$, where $\mathbf{0}$ is an $N \times 1$ vector with all entries equal to 0 . There are two stages in each iteration. In the first stage, an optimal solution $\mathbf{b}^{i \star}$ is computed by solving the following problem,

$$
\begin{array}{r}
\min _{\mathbf{b}^{i}}\left\|\hat{\mathbf{A}} \mathbf{b}^{i}\right\|^{2} \\
\text { s.t. }+\operatorname{Im}\left(s_{k}^{*}\left(\tilde{\mathbf{h}}_{k}+\mathbf{e}_{k}\right)^{\top} \hat{\mathbf{A}} \mathbf{b}^{i}\right) \leq \\
\left(\operatorname{Re}\left(s_{k}^{*}\left(\tilde{\mathbf{h}}_{k}+\mathbf{e}_{k}\right)^{\top} \hat{\mathbf{A}} \mathbf{b}^{i}\right)-\gamma_{k}\right) \tan \theta, \\
\forall \mathbf{e}_{k} \in \mathcal{E}_{k}^{i+}, \forall k \in \mathcal{K}, \\
-\operatorname{Im}\left(s_{k}^{*}\left(\tilde{\mathbf{h}}_{k}+\mathbf{e}_{k}\right)^{\top} \hat{\mathbf{A}} \mathbf{b}^{i}\right) \leq \\
\left(\operatorname{Re}\left(s_{k}^{*}\left(\tilde{\mathbf{h}}_{k}+\mathbf{e}_{k}\right)^{\top} \hat{\mathbf{A}} \mathbf{b}^{i}\right)-\gamma_{k}\right) \tan \theta, \\
\forall \mathbf{e}_{k} \in \mathcal{E}_{k}^{i-}, \forall k \in \mathcal{K},
\end{array}
$$

where the constraint in (6b) enforces the received signal to lie below the anti-clockwise decision boundary, and the constraint in (6c) enforces the received signal to lie above the clockwise decision boundary as illustrated in Fig. 4. This optimization problem is a quadratic problem with finite linear constraints, thus, it can be efficiently solved with CVX.

In the second stage, the worst-case error vectors at $\mathbf{b}=$ $\mathbf{b}^{i \star}, \forall k \in \mathcal{K}$, are computed. The worst-case error vector $\mathbf{e}_{k}^{i+}$ of constraint (6b) is defined as an error vector $\mathbf{e} \in \mathcal{E}$ that violates the constraint (6b) with the largest margin, or fulfills it with the smallest margin when $\mathbf{b}=\mathbf{b}^{i \star}$ [11]. The worst-case error vector $\mathbf{e}_{k}^{i-}$ of constraint (6c) can be defined in a similar way for the clockwise drift of the received signal. By equating the derivatives of the constraints (6b) and (6c) to zero, the closed-form expressions of the elements of the worst-case error vectors for the problem (5) for the channel quantization error model described by Eq. (2) can be computed as

$$
\begin{gathered}
e_{k n}^{i+}=\frac{d}{2} \operatorname{sign}\left(\operatorname{Im}\left(z_{k n}\right)-\operatorname{Re}\left(z_{k n}\right) \tan \theta\right)+ \\
j \frac{d}{2} \operatorname{sign}\left(\operatorname{Re}\left(z_{k n}\right)+\operatorname{Im}\left(z_{k n}\right) \tan \theta\right), \\
e_{k n}^{i-}=\frac{d}{2} \operatorname{sign}\left(-\operatorname{Im}\left(z_{k n}\right)-\operatorname{Re}\left(z_{k n}\right) \tan \theta\right)+ \\
j \frac{d}{2} \operatorname{sign}\left(-\operatorname{Re}\left(z_{k n}\right)+\operatorname{Im}\left(z_{k n}\right) \tan \theta\right),
\end{gathered}
$$

where $\mathbf{z}_{k} \triangleq s_{k}^{*} \hat{\mathbf{A}} \mathbf{b}^{i \star}$. Now, if either of the worst-case error vectors violates the corresponding constraint, it will be added to the corresponding set of error vectors, i.e.,

$$
\begin{aligned}
\mathcal{E}_{k}^{(i+1)+}=\mathcal{E}_{k}^{i+} \cup \mathbf{e}_{k}^{i+}, & \forall k \in \mathcal{K}, \\
\mathcal{E}_{k}^{(i+1)-}=\mathcal{E}_{k}^{i-} \cup \mathbf{e}_{k}^{i-}, & \forall k \in \mathcal{K} .
\end{aligned}
$$

When all worst-case error vectors satisfy the corresponding constraints, we conclude that the solution of problem (6) is the global optimal solution and thus terminate the algorithm [11].

For the channel estimation error model described by Eq. (3) the closed-form expressions of the elements of the worst-case error vectors are given by

$$
\begin{gathered}
e_{k n}^{i+}=\frac{\lambda}{\left|z_{k n}\right|}\left(\operatorname{Im}\left(z_{k n}\right) \cos \theta-\operatorname{Re}\left(z_{k n}\right) \sin \theta\right)+ \\
j \frac{\lambda}{\left|z_{k n}\right|}\left(\operatorname{Re}\left(z_{k n}\right) \cos \theta+\operatorname{Im}\left(z_{k n}\right) \sin \theta\right), \\
e_{k n}^{i-}=\frac{\lambda}{\left|z_{k n}\right|}\left(-\operatorname{Im}\left(z_{k n}\right) \cos \theta-\operatorname{Re}\left(z_{k n}\right) \sin \theta\right)+ \\
j \frac{\lambda}{\left|z_{k n}\right|}\left(-\operatorname{Re}\left(z_{k n}\right) \cos \theta+\operatorname{Im}\left(z_{k n}\right) \sin \theta\right) .
\end{gathered}
$$

\section{SIMULATION RESULTS}

In this section, we compare the performance of the proposed robust hybrid precoding approach with the non-robust design for both channel error models. In the simulation, a co-channel multi-user massive MIMO downlink system is considered, where the BS is equipped with $N=64$ antennas and $8 \mathrm{RF}$ chains, and serves 8 single-antenna users. In each transmission block, 8 symbols are drawn from the BPSK/QPSK constellation and the threshold margin is assumed to be the same for all the users, i.e., $\Gamma_{k}=\Gamma, \forall k \in \mathcal{K}$. The codebookbased BMCS method is applied to compute the analog precoding matrix $\mathbf{A}$, where the element of the codebook matrix $\mathbf{C}=\left[\mathbf{c}_{1}, \ldots, \mathbf{c}_{N}\right]$ has a constant magnitude $1 / \sqrt{N}$, such that $\left\|\mathbf{c}_{n}\right\|=1$. The noise at each user is assumed to be i.i.d. AWGN with unit variance, i.e., $n_{k} \sim \mathcal{C N}(0,1)$. The simulation results are averaged over 5000 Monte-Carlo iterations.

Figs. 5 and 6 illustrate the achieved SER for channel quantization error and channel estimation error, respectively. The 


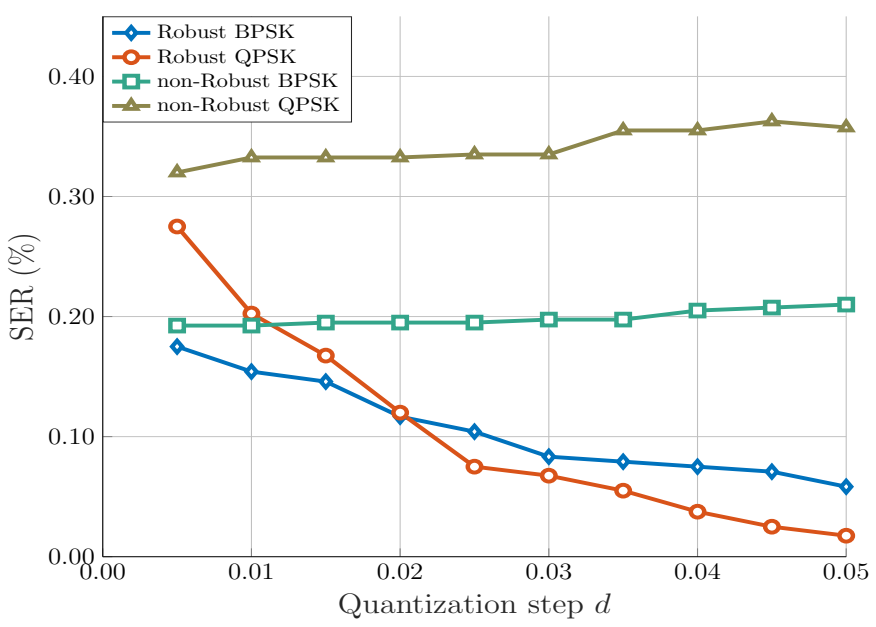

Fig. 5. SER vs. quantization step $d$ with $\Gamma=1$, TNR $=4$ for channel quantization error model.

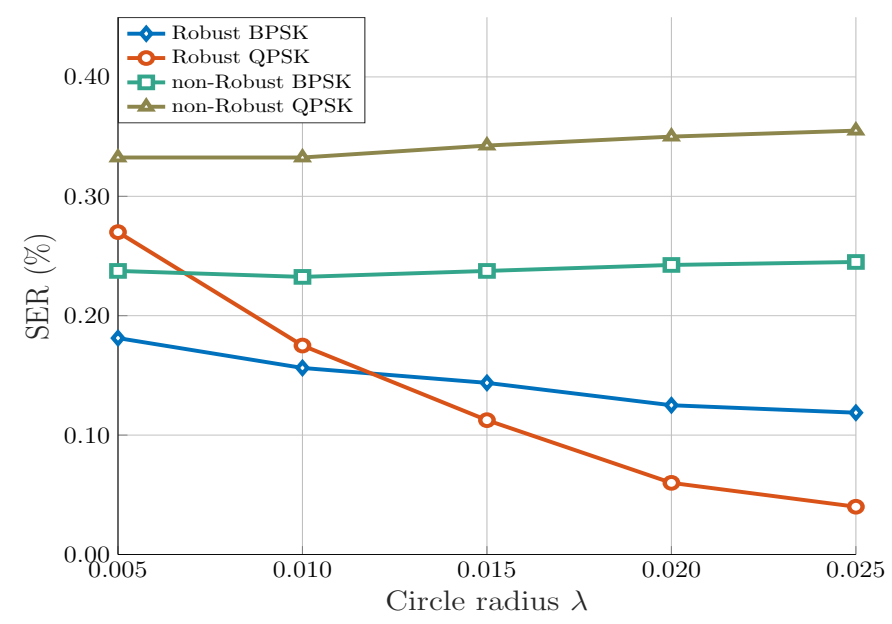

Fig. 6. SER vs. circle radius $\lambda$ with $\Gamma=1$, TNR $=4$ for channel estimation error model.

threshold margin-to-noise power ratio (TNR) is set with TNR $\triangleq \Gamma_{k} / \sigma_{k}^{2}=4$, which measures the relative margin between the CI-region and the corresponding decision boundaries with respect to the noise power and directly affects the achieved SER. The quantization step $d$ is chosen in $0.005 \sim 0.05$, which is approximately in the range of the precision of the codebook represented with $4 \sim 7$ digits $^{2}$. It can be observed that the proposed robust hybrid precoding scheme achieves a lower SER for BPSK and QPSK modulations in both channel error models when comparing to the non-robust design. Especially when the channel error increases, the achieved SER of our robust hybrid precoding decreases significantly. However, such improved performance of our robust hybrid precoding comes at the expense of increased transmit power for the channel quantization error model and channel estimation

\footnotetext{
${ }^{2} \mathrm{~A}$ codebook represented with $n$ digits has a precision of $1 / 2^{n}$.
}

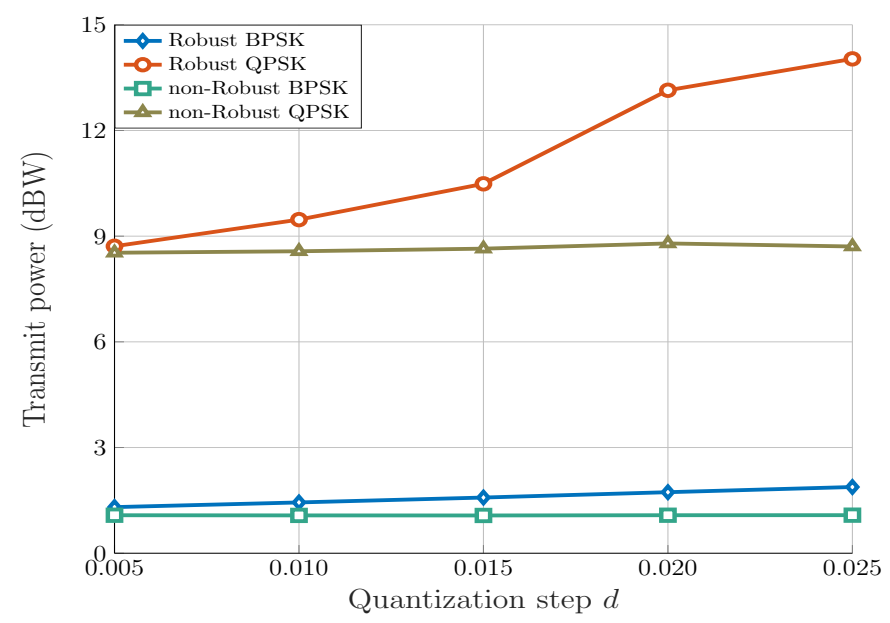

Fig. 7. Average transmit power vs. quantization step $d$ with $\Gamma=1$ for channel quantization error model.

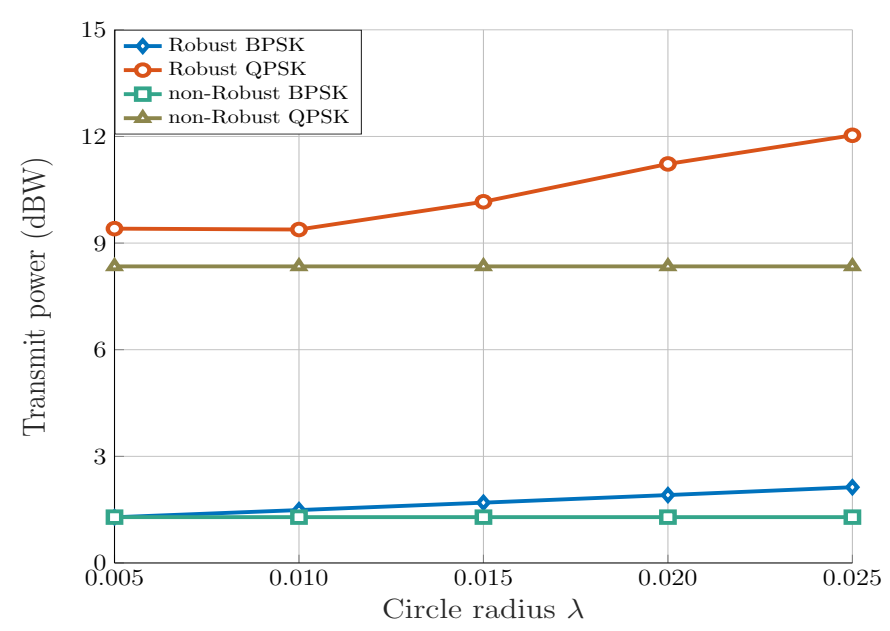

Fig. 8. Average transmit power vs. circle radius $\lambda$ with $\Gamma=1$ for channel estimation error model.

error model when compared to that of non-robust design as illustrated in Figs. 7 and 8, respectively.

\section{CONCLUSION}

Multi-user massive MIMO downlink systems suffering from channel quantization errors and channel estimation errors are considered. A robust CI-based hybrid precoding approach is proposed, where the hybrid precoding is decomposed into analog precoding and robust digital precoding. The closedform expressions of the worst-case error vectors are derived to apply the iterative algorithm in computing the robust digital precoder. We compared the performance of the proposed robust hybrid precoding with the non-robust design for both channel error models. The simulation results show that the robust design achieves a lower SER at an expense of higher transmit power. 


\section{REFERENCES}

[1] C. H. Doan et al., "Design Considerations for $60 \mathrm{GHz}$ CMOS Radios," IEEE Commun. Mag., vol. 42, no. 12, pp. 132-140, Dec. 2004

[2] E. Björnson, E. G. Larsson, and T. L. Marzetta, "Massive MIMO: Ten Myths and One Critical Question," IEEE Commun. Mag., vol. 54, no. 2, pp. 114-123, Feb. 2016.

[3] H. Jakob, S. ten Brink, and M. Debbah, "Massive MIMO in the UL/DL of Cellular Networks: How Many Antennas Do We Need?," IEEE J. Select. Areas Commun., vol. 31, no. 2, pp. 160 - 171, Feb. 2013.

[4] L. Liang, W. Xu, and X. Dong, "Low-Complexity Hybrid Precoding in Massive Multiuser MIMO Systems," IEEE Wireless Commun. Letters, vol. 3, no. 6, pp. 653-656, Dec. 2014.

[5] F. Sohrabi and W. Yu, "Hybrid Digital and Analog Beamforming Design for Large-Scale Antenna Arrays," IEEE J. Select. Topics in Signal Process., vol. 10, no. 3, pp. 501-513, Apr. 2016.

[6] X. Zhang, A. F. Molisch, and S. Kung, "Variable-Phase-ShiftBased RF-Baseband Codesign for MIMO Antenna Selection," IEEE Trans. Signal Process., vol. 53, no. 11, pp. 4091-4103, Nov. 2005.

[7] Z. Li et al., "Joint Optimization of Hybrid Beamforming for Multi-User Massive MIMO Downlink," IEEE Trans. Wireless Commun., vol. 17, no. 6, pp. 3600-3614, Jun. 2018.

[8] A. F. Molisch et al., "Hybrid Beamforming for Massive MIMO: A Survey," IEEE Commun. Mag., vol. 55, no. 9, pp. 134-141, Sep. 2017.

[9] G. Hegde, Y. Cheng, and M. Pesavento, "Hybrid Beamforming for Large-Scale MIMO Systems Using Uplink-Downlink Duality," in Proc. IEEE Int. Conf. on Acoustics, Speech and Signal Process. (ICASSP), Mar. 2017, pp. 3484-3488.

[10] F. Sohrabi and W. Yu, "Hybrid Analog and Digital Beamforming for mmWave OFDM Large-Scale Antenna Arrays," IEEE J. Select. Areas Commun., vol. 35, no. 7, pp. 1432-1443, Jul. 2017.

[11] G. Hegde, C. Masouros, and M. Pesavento, "Interference Exploitation-Based Hybrid Precoding with Robustness Against Phase Errors," IEEE Trans. Wireless Commun., vol. 18, no. 7, pp. 3683-3696, Jul. 2019.

[12] G. Zheng et al., "Rethinking the Role of Interference in Wireless Networks," IEEE Commun. Mag., vol. 52, no. 11, pp. 152-158, Nov. 2014.

[13] M. Alodeh, S. Chatzinotas, and B. Ottersten, "Constructive Multiuser Interference in Symbol Level Precoding for the MISO Downlink Channel," IEEE Trans. Signal Process., vol. 63, no. 9, pp. 2239-2252, May 2015.

[14] C. Masouros and G. Zheng, "Exploiting Known Interference as Green Signal Power for Downlink Beamforming Optimization,” IEEE Trans. Signal Process., vol. 63, no. 14, pp. 36283640, Jul. 2015.

[15] S. Timotheou et al., "Exploiting Constructive Interference for Simultaneous Wireless Information and Power Transfer in Multiuser Downlink Systems," IEEE J. Select. Areas Commun., vol. 34, no. 5, pp. 1772-1784, May 2016.
[16] C. Masouros et al., "Known Interference in the Cellular Downlink: A Performance Limiting Factor or A Source of Green Signal Power?," IEEE Commun. Mag., vol. 51, no. 10, pp. 162-171, Oct. 2013.

[17] R. Vannithamby and S. Talwar, Towards 5G: Applications, Requirements and Candidate Technologies, John Wiley \& Sons, 2017.

[18] E. Björnson, D. Hammarwall, and B. Ottersten, "Exploiting Quantized Channel Norm Feedback Through Conditional Statistics in Arbitrarily Correlated MIMO Systems," IEEE Trans. Signal Process., vol. 57, no. 10, pp. 4027-4041, Oct. 2009.

[19] J. Li et al., "Robust and Low Complexity Hybrid Beamforming for Uplink Multiuser MmWave MIMO Systems," IEEE Commun. Letters, vol. 20, no. 6, pp. 1140-1143, Jun. 2016.

[20] H. Seleem, A. I. Sulyman, and A. Alsanie, "Hybrid Precoding-Beamforming Design with Hadamard RF Codebook for mmWave Large-Scale MIMO Systems," IEEE Access, vol. 5, pp. 6813-6823, Jun. 2017.

[21] A. Liu and V. K. N. Lau, "Impact of CSI Knowledge on the Codebook-Based Hybrid Beamforming in Massive MIMO," IEEE Trans. Signal Process., vol. 64, no. 24, pp. 6545-6556, Dec. 2016.

[22] W. Tan et al., "Spectral and Energy Efficiency of Massive MIMO for Hybrid Architectures Based on Phase Shifters," IEEE Access, vol. 6, pp. 11751-11759, Mar. 2018.

[23] X. Zhu et al., "Adaptive Hybrid Precoding for Multiuser Massive MIMO," IEEE Commun. Letters, vol. 20, no. 4, pp. 776779, Apr. 2016.

[24] T. L. Marzetta, "Noncooperative Cellular Wireless with Unlimited Numbers of Base Station Antennas," IEEE Trans. Wireless Commun., vol. 9, no. 11, pp. 3590-3600, Nov. 2010.

[25] L. Lu et al., "An Overview of Massive MIMO: Benefits and Challenges," IEEE J. Select. Topics in Signal Process., vol. 8, no. 5, pp. 742-758, Oct. 2014.

[26] K. L. Law, I. Wajid, and M. Pesavento, “Optimal Downlink Beamforming for Statistical CSI with Robustness to Estimation Errors," Signal Process., vol. 131, no. C, pp. 472-482, Feb. 2017.

[27] B. Sklar and F. J. Harris, Digital Communications: Fundamentals and Applications, vol. 2001, Prentice-hall Englewood Cliffs, NJ, 1988.

[28] E. Karipidis, N. D. Sidiropoulos, and Z. Luo, "Quality of Service and Max-Min Fair Transmit Beamforming to Multiple Cochannel Multicast Groups," IEEE Trans. Signal Process., vol. 56, no. 3, pp. 1268-1279, Mar. 2008.

[29] S. Boyd and L. Vandenberghe, Convex Optimization, Cambridge university press, 2004.

[30] G. Hegde, C. Masouros, and M. Pesavento, "Analog Beamformer Design for Interference Exploitation Based Hybrid Beamforming," in Proc. IEEE Sensor Array and Multichannel Signal Process. Workshop (SAM), Jul. 2018, pp. 109-113.

[31] A. Alkhateeb, G. Leus, and R. W. Heath, "Limited Feedback Hybrid Precoding for Multi-User Millimeter Wave Systems," IEEE Trans. Wireless Commun., vol. 14, no. 11, pp. 64816494, Nov. 2015. 